\title{
Satiation effects in continuous reinforcement and successive sensory discrimination situations*
}

\author{
IRMINGARD I. LENZER and CAROL A. WHITE \\ Saint Mary's University, Halifax, Nova Scotia, Canada
}

\begin{abstract}
Nine rats with forebrain and hypothalamic electrodes were trained on a sensory discrimination task with $\mathrm{S}-$ intervals ranging from 12 to $60 \mathrm{sec}$. Their performance during a 48-h discrimination session was compared to that during a 48-h $\mathrm{CRF}$ session. The response measures showed that forebrain Ss performed more poorly than hypothalamic Ss during the discrimination session, even though performance of forebrain animals was better in the discrimination session than in the CRF session when time until the first 5-min pause was considered. In the CRF session, there was no difference in performance of forebrain and hypothalamic animals, except in terms of time till first pause. The results are discussed in terms of cumulative effects of forebrain and hypothalamic ESB.
\end{abstract}

Studies dealing with behavior reinforced by rewarding electrical stimulation of the brain (ESB) show differences in behavior as a function of electrode placement. For example, in an operant situation with the animals responding on a continuous reinforcement schedule (CRF), there are differences in the short-term response rate depending on whether the electrode is in the forebrain or in the lateral hypothalamus. Olds (1958), comparing the performance of hypothalamic and forebrain Ss over the course of a 48-h session with ESB available on a CRF schedule, found that animals with forebrain electrodes responded more slowly after 4 to $8 \mathrm{~h}$, whereas hypothalamic Ss responded at a high steady rate for longer periods of time, until overcome by "physical exhaustion." Olds also found that hypothalamic Ss returned, during at least $1 \mathrm{~h}$ of the second day of the prolonged session, to their original high rates of responding, but that forebrain animals did not return to their initial rates. One possible explanation of the data on short-term rate is that the forebrain ESB differs from the hypothalamic ESB in that it more readily produces seizures or some other kind of interfering side effect. The satiation effect would need the additional assumption that the forebrain ESB produces a cumulative or long-term increase in the frequency of these side effects. It would follow from this analysis that if the frequency or intensity of the interfering side effects could be reduced by some experimental manipulation, then the differences between the forebrain and the hypothalamic animals could be reduced. With respect to the question of short-term rate, Reid, Gibson, Gledhill, and Porter (1964) found that the administration of an

*This research was supported in part by a United States Mental Health grant to Dr. G. Frommer, Indiana University, Bloomington, Indiana, and a National Research Council of Canada grant to I. Lenzer. The authors are grateful to Dr. G. Frommer for financial assistance and for suggestions during the early stages of the experiment. anticonvulsant drug and the consequent elevation of the seizure threshold had the effect of increasing the response rate of animals responding for forebrain ESB, to levels more nearly comparable to those displayed by hypothalamic animals. With respect to the question of satiation, Atrens (1970) found that forebrain animals trained to respond on a VI $30-\mathrm{sec}$ schedule with five ESBs per reinforcement period responded for long periods of time, up to $12-17 \mathrm{~h}$, without pausing for more than $5 \mathrm{~min}$ at a time; the use of the VI schedule, which reduces the frequency or density of the ESBs and therefore also presumably the interfering side effects, seems to have reduced the satiation effect in forebrain animals and made the forebrain animals more nearly comparable to the hypothalamic animals in this respect.

Lenzer and Frommer $(1968,1971)$ found that forebrain animals trained to make a successive sensory - discrimination with one ESB train per S+ period perform as well as, or nearly as well as, hypothalamic animals trained on the same task. Again, it might be assumed that this result is due to the fact that in the discrimination situation, as compared to the CRF situation, the reinforcement density is lower and as a consequence so is the frequency or intensity of the interfering side effects. The present experiment was done to compare the performance of hypothalamic and forebrain animals over the course of a 48-h session of discrimination responding and also over the course of a 48-h session of CRF responding. The hypothesis was that the forebrain animals would show a greater satiation effect in the CRF session than would the hypothalamic animals, but that this difference would be reduced or eliminated in the discrimination session because of the lower reinforcement density and the consequent decrease in the frequency or intensity of the interfering side effects.

\section{METHOD}

The apparatus for the present experiment was the same as that 
used in the previous studies (Lenzer \& Frommer, 1968, 1971). The S+ consisted of the light from a No. 313 lamp, set in an amber jewel 3 in. above the operant lever, together with white noise, coming from a white-noise source outside the experimental chamber and having a 63-dB SPL inside the chamber. The $\mathrm{S}$ - was defined to be the absence of the $\mathrm{S}+$. The program controlling the stimulus events was designed so that, in the discrimination situation, the animal had to cease responding during the $S$ - period for at least $10 \mathrm{sec}$ before the scheduled onset of the S+; this was to facilitate the learning of the discrimination. Another feature of the program was that if the animal failed to respond to the $\mathrm{S}+$ within $58 \mathrm{sec}$, the $\mathrm{S}+$ was terminated.

The Ss were male albino Wistar rats, about 90-120 days of age and weighing about $350 \mathrm{~g}$ at the beginning of the experiment. All animals received food and water ad lib throughout the experiment. Permanent bipolar electrodes (Lenzer \& Frommer, 1968), aimed at the left septal area (coordinates: $2-3 \mathrm{~mm}$ anterior to bregma, $1 \mathrm{~mm}$ lateral to the midline of the skull, and $6.5 \mathrm{~mm}$ below the brain surface) and the left hypothalamic area (coordinates: $3.5 \mathrm{~mm}$ posterior to bregma, $1 \mathrm{~mm}$ lateral to the midline of the skull, and $9.5 \mathrm{~mm}$ below the brain surface), were implanted in each animal under pentobarbital (Nembutal) anesthesia $(50 \mathrm{mg} / \mathrm{kg}$, IP).

Following a 3-day recovery period, each animal was trained to press a lever for ESB reward in the presence of the S+. Following the initial shaping period, which lasted for 1 or 2 days, each animal was kept on a CRF (continuous reinforcement) schedule for 3 days, each daily session lasting about $1 \mathrm{~h}$. The animals were assigned randomly to either the septal or hypothalamic group, and that electrode was used. However, if the electrode site did not support self-stimulation, the second electrode was used. During the CRF phase, the ESB parameters were fixed: The waveform was biphasic rectangular, the pulse width was $0.5 \mathrm{msec}$, the interval between the positive and the negative pulses of each pulse pair was $5 \mathrm{msec}$, there were 100 pulse pairs/second, the train duration was $300 \mathrm{msec}$; stimulus intensity was adjusted for each animal individually (see Table 1), ranging from 350 to 1,000 microA. The highest stimulus intensity which would support barpressing without motor side effects was chosen. This was done in order to ensure seizures during CRF, given that the electrode was in a seizure-prone area. Reduction in density of ESBs in the discrimination situation should improve the behavior of the septal animals if indeed overt or subliminal seizures prevented the animals from performing as well as the hypothalamic Ss. All septal Ss did, in fact, exhibit overt seizures during the CRF phase.

The animals were then taught to discriminate the $S+$ from the $\mathrm{S}-$. The $\mathrm{S}+$ period lasted as long as it took the animal to make 31 leverpresses, each leverpress being reinforced with one ESB burst, or train. The discrimination learning phase lasted from 4 to 6 days, each daily session lasting about $1 \mathrm{~h}$. The $S-$ period was gradually increased in duration from $15 \mathrm{sec}$ on the first day to $30 \mathrm{sec}$ on the second and $1 \mathrm{~min}$ on the third day. On the fourth day, the animal was presented with a random sequence of $15-, 30-$, and 60-sec $S$ - periods, with the $S+$ lasting as long as it took the animal to make one response within the 58 -sec limit. For some animals, this procedure was repeated on the next day if the animal failed to respond to the $\mathrm{S}+$ on several trials.

On the following day, the 48-h discrimination session began. The programming tape, which controlled the onset of the S+, had a random sequence of 12-, 24-, 48-, 54-, and 60-sec Speriods.

On the third day after cessation of the discrimination session, the animal was placed on a CRF schedule and allowed to leverpress for $1 \mathrm{~h}$. On the sixth day after the discrimination session, the animal was placed on a CRF schedule and allowed to respond for $48 \mathrm{~h}$. The $48 \mathrm{~h}$ discrimination and CRF sessions started for each animal at noon. The animal had access to wet mash and water in the box for the entire session, and was left unattended for a large part of the session.
Several days after the end of the CRF session, the second electrode was tested. If it supported self-stimulation, the animal received identical treatment as for the first electrode, i.e., it started with Day 1 of CRF training. Three Ss $(478,481$, and 482) fall into this category. S 478 had its first run on the hypothalamus, followed by the septum; 481 and 482 were first run on the septum. For three hypothalamic Ss, no data were collected for the last 4-6 h of the 48-h discrimination session. In one case, the cord became disconnected, and in the other two cases, the pen recorder for the discrimination broke down.

At the end of the experiment, all animals were sacrificed and perfused with $0.9 \%$ saline, followed by $10 \%$ formalin in $0.9 \%$ saline solution. Sections of the extracted brains were frozen and sliced at 40 microns. They were stained with cresyl violet.

\section{RESULTS}

The electrode tips were located in the hypothalamus and the forebrain. The hypothalamic tips for Ss 484 and 478 could not be precisely located due to tearage of brain tissue. The electrode locations are given for each animal in Table 1.

\section{8-h CRF Session}

The data for the 48-h CRF session were analyzed according to the Olds (1958) method, expressing the highest hourly rate in the second $24 \mathrm{~h}$ as a percentage of the rate during the first hour; Olds called this percentage the "endurance score." From Table 1, Column 6, it can be seen that only one hypothalamic animal (S 480) improved its performance during the second 24-h period with respect to its rate during the first hour, whereas three out of five forebrain animals $(478,481$, and 148) improved their performance. The high endurance scores for the forebrain group reflect the fact that some of the forebrain animals responded at a relatively low rate for the first hour or two of the session and only later attained a maximum rate; Ss 478,481 , and 148 showed an increase in rate of response of $37 \%, 88 \%$, and $176 \%$, respectively, over the first $5 \mathrm{~h}$ of the session. The hypothalamic Ss, on the other hand, showed their highest response rate during the first hour, except for two animals that responded slightly faster during the second hour.

To allow for improvement in rate of response during the first few hours of the session as well as to avoid hourly response rates reflecting transient increases or decreases in responding, the endurance score was calculated from the mean of the $3 \mathrm{~h}$ with the highest response rate during the first $5 \mathrm{~h}$ of the session (mean initial rate, Table 1, Column 4) and the mean of the $3 \mathrm{~h}$ with the highest response rates during the second 24-h period; the latter was then expressed as a percentage of the mean initial rate. These newly calculated endurance scores are shown in Table 1, Column 5. It is apparent that the redefinition of the endurance scores did not change the scores considerably for the hypothalamic group but did so for the forebrain group. The endurance scores do not differ significantly for the two groups (the 
Table 1

Initial Performance and Endurance Scores for Hypothalamic and Forebrain Animals in CRF and Discrimination Session

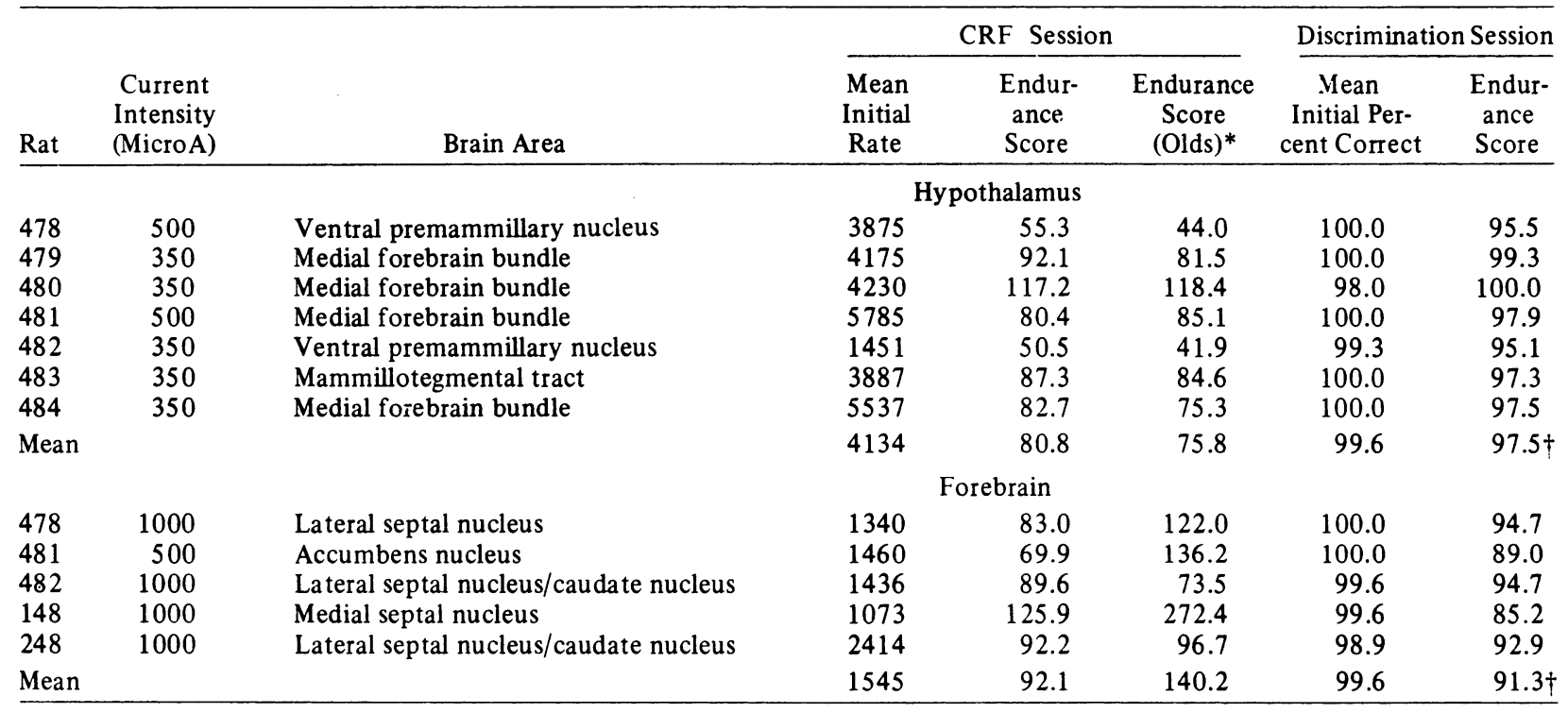

*Endurance score as calculated by the Olds (1958) method (see Results section).

†Difference between means is significant at $p=.002$, two-tailed.

Mann-Whitney $\mathrm{U}$ test yielded $\mathrm{p}>.10$ ).

Since the endurance score reflects the behavior of the animal only during a few hours of the 48-h session, decrement of performance was assessed for the total session. The 48-h session was divided into blocks of $6 \mathrm{~h}$. For each animal, the mean response rate of a given 6-h block was calculated as a percentage of the mean initial rate (as defined above); these percentage scores for each 6-h block are called "mean percent decrements." Mean percent decrements for the entire CRF session are shown in Fig. 1, in the form of averages for the forebrain and the hypothalamic group. The Mann-Whitney U test showed that, for each 6-h block, the differences between the mean percent decrements for the two groups were not significant.
A further analysis was performed to determine whether hypothalamic and forebrain animals differed in the time of occurrence of the first pause in responding of $5 \mathrm{~min}$ or longer. It was found that the mean onset of the first pause was in the 13th hour for the hypothalamic Ss and in the 4th hour for the forebrain Ss. The difference between these means is significant (Mann-Whitney $U$ test: $U=2, p=.01$, two-tailed).

\section{8-h Discrimination Session}

All animals learned the discrimination, as indicated by short-latency responses to the onset of the S+, with virtually no responding during the $\mathrm{S}-$ interval. Long-term performance in the discrimination situation
Fig. 1. Mean percent decrement of responses for each 6-h block over the 48-h CRF and discrimination session for hypothalamic and forebrain Ss. *The mean for Hours 43-48 (Hyp. Disc.) includes data for only four of the seven hypothalamic Ss.

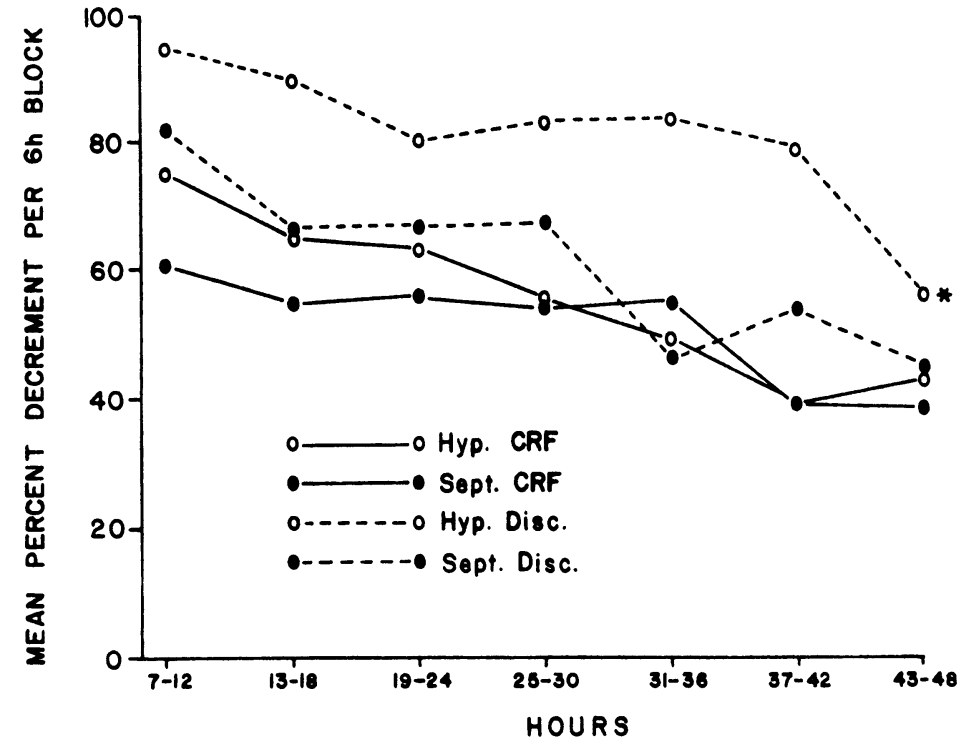


was evaluated in the following way. For each hour, the number of times the S+ came on was counted and the latency of response to each S+ was recorded. A "correct" response was defined as a response within $10 \mathrm{sec}$ or less. Latencies longer than $10 \mathrm{sec}$, or no response to the $\mathrm{S}+$ at all, were defined as incorrect. For each hour, the total number of correct responses was expressed as a percentage of the total number of $\mathrm{S}+$ presentations in that hour. A score of 100 means that the animal responded to all $\mathrm{S}+$ presentations with a latency of $10 \mathrm{sec}$ or less. An endurance score was then calculated for each animal; the mean of the percent correct of the highest $3 \mathrm{~h}$ in the second $24-\mathrm{h}$ period was expressed as a percentage of the mean of the $3 \mathrm{~h}$ with the highest percent correct during the first $5 \mathrm{~h}$ of the session (the "mean initial percent correct"). From Table 1, Column 7, it can be seen that forebrain Ss performed just as well as hypothalamic Ss during the first $5 \mathrm{~h}$ of the session, in terms of the mean initial percent correct. However, the endurance scores (Table 1, Column 8) for the hypothalamic group are significantly higher than those for the forebrain group $(U=0, p=$ .002 , two-tailed).

A further analysis was done to determine whether the lowest endirance score ( $85.2 \%$ of $S$ 148) reflected an increase in response latency to the $\mathrm{S}+$ or a failure to respond to the $\mathrm{S}+$. Response latencies to the $\mathrm{S}+$ for the $6 \mathrm{~h}$ that made up the endurance score were tabulated. The mean response latency for the first 3 highest hours was $3.6 \mathrm{sec}(3.3,3.4$, and $4.1 \mathrm{sec}$ for the $3 \mathrm{~h}$, respectively) with no failures to respond to the S+. For the highest $3 \mathrm{~h}$ in the second 24-h period, the mean response latency to the $\mathrm{S}+$ on trials on which $\mathrm{S}$ responded to the $S+$ was $4.6 \mathrm{sec}(3.1,4.1$, and $6.3 \mathrm{sec}$ for each of the $3 \mathrm{~h}$ ). During these $3 \mathrm{~h}$, the $\mathrm{S}$ failed to respond to the $\mathrm{S}+29$ times. These data suggest that the low endurance score results not from an increase in latency of response but from an increase in the number of no responses; when an animal does respond during the second $24 \mathrm{~h}$, or at least during the highest $3 \mathrm{~h}$ of the second $24 \mathrm{~h}$, it responds just as fast as it does during the first few hours of the session. Visual inspection of data for other animals supports this conclusion.

To get an overall picture of the performance of the animals in the discrimination situation, the mean percent correct responses for each 6-h block was calculated for the forebrain and the hypothalamic group; these means are shown in Fig. 1. For each 6-h block, a Mann-Whitney $\mathrm{U}$ test was done to compare the performance of hypothalamic and forebrain animals. With two exceptions (Hours 19-24 and 43-48), the mean percent correct responses was significantly higher for the hypothalamic group (Hours 7-12: $U=4, p=.03$; Hours 13-18: $U=6, p=.07$; Hours 19-24: $U=23$, $\mathrm{p}>.10$; Hours 25-30: $U=0, p=.002$; Hours 31-36: $U=$ $3, \mathrm{p}=.018$; Hours $37-42: \mathrm{U}=6, \mathrm{p}=.07$; Hours 43-48: $\mathrm{U}$ $=8, \mathrm{p}>.10$; all tests are two-tailed).

\section{DISCUSSION}

The data fail to substantiate the original hypothesis. On the contrary, the differences between the forebrain and the hypothalamic animals were greater in the long discrimination session than in the long CRF session. This result suggests that some factor other than interfering side effects is at work.

Two factors may account for the failure to replicate the Olds study. First of all, the response rates of the Ss in the present experiment were higher than the rates in the Olds study; the mean response rate of the hypothalamic Ss in the Olds experiment was 1,343 ; for the forebrain Ss, it was 882 . In the present experiment, the rates were 4,134 and 1,545 for the two groups, respectively. According to Olds (1958), the relatively low rate of the hypothalamic Ss was due to the fact that Ss that responded very fast became disconnected from the electrical circuit due to rapid turning and twisting of the cord. From this, it may be argued that animals that leverpress very rapidly, as in the present experiment, show a greater decrement due to physical exhaustion than do animals with response rates in the range reported by Olds. A second explanation may be the high current intensity used for the forebrain ESB in the present experiment. Ray, Hine, and Bivens (1968) found that with preoptic electrodes, animals showed less response decrement with high-intensity ESB than with low-intensity ESB.

However; forebrain Ss did show poorer performance, compared to hypothalamic Ss, when onset of first pause in excess of $5 \mathrm{~min}$ was considered. Forebrain Ss paused significantly earlier in the session than did hypothalamic Ss. There are two possible explanations to be considered. First of all, the animals may have stopped responding because of a seizure or because they were avoiding a seizure. However, while seizures are often the consequence of forebrain ESB, the evidence suggests that they do not account for the pauses after several hours of a prolonged session; Herberg, Tress, and Blundell (1969) have shown that seizures occur more frequently at the very beginning of the session than later on in the session. The second possibility is that these pauses may simply be rest periods after hours of hard work. That hypothalamic Ss show pauses later in the session may mean that ESB to the hypothalamus arouses the animal or facilitates locomotor behavior, as Christopher and Butter (1968) have shown, with the result that hypothalamic Ss stop responding later, when fatigue overcomes this arousing or facilitatory effect of the ESB.

The mean of the initial percent correct responses in the discrimination situation was identical for the two groups. In this respect, the hypothesis that performance of forebrain animals can be made to approximate that of hypothalamic animals by reducing reinforcement density is borne out. 
Atrens (1970) reported that forebrain Ss improved performance on a long session with a VI-30 schedule and five ESBs per VI interval. His forebrain animals showed stable behavior (as defined by lack of pauses in excess of $5 \mathrm{~min}$ ) from 12 to $17 \mathrm{~h}$ in comparison to the more cyclical behavior observed on the CRF schedule. The present data support this finding in one respect: the first pause in excess of $5 \mathrm{~min}$ occurred later in the discrimination than in the CRF session. However, the data also show that although the performance of the forebrain Ss was better, in terms of time to first pause, in the discrimination session than in the CRF session, it was nevertheless inferior to that of the hypothalamic Ss on all three measures, endurance scores, percent decrement scores, and time till first pause; in fact, it was more clearly inferior in the discrimination session than in the CRF session. Atrens (1970) himself did not run a hypothalamic control group and thus had no basis to conclude that forebrain ESB was "motivationally homologous" to hypothalamic ESB.

There are several possible explanations of the finding that forebrain animals showed significantly lower endurance and percent decrement scores in the discrimination situation than did hypothalamic animals. First, it may be argued that forebrain Ss, even though they received only one ESB per S+, still experienced seizures because of the high current intensity. However, if the occurrence of seizures prevented the animals from responding to the $\mathrm{S}+$, it would be expected that this effect should have manifested itself particularly.in the initial hours of the session, when seizures occur most frequently (Herberg et al, 1969). But it was during the initial hours of the session that forebrain Ss performed as well as the hypothalamic Ss.

A second possibility, based on the conflict hypothesis of brain stimulation (Kent \& Grossman, 1969), is that the forebrain ESB became more ambiguous over time. However, with this hypothesis, it would be difficult to explain the findings of the CRF session where the animal collects a large number of ESBs; the differences (between forebrain and hypothalamic animals) should be as large as, if not larger than, those in the discrimination situation. Also, the conflict hypothesis assumes that the aversive component becomes stronger over a period of no responding; this would result in a failure to initiate a response after a long pause. Yet, forebrain Ss in both the CRF and the discrimination session did return after long pauses to the lever without priming. A third finding which argues against a conflict interpretation concerns the short-latency responses (see latency analysis of the endurance score for S 148) to the St. If an. approach-avoidance conflict existed in the animal, then it would manifest itself in an increased latency of response; but the fact is that when the animal did respond during the highest $3 \mathrm{~h}$ of the second day, it responded with short latencies.

A third possibility rests on the experimental findings that stimulation of certain areas in the forebrain, in particular the septum, produces inhibition of neurons that respond to peripheral stimulation (Ball, 1967; Dafney \& Feldman, 1969). The discrimination schedule, in contrast to the VI schedule with short intervals (Atrens, 1970) or the CRF schedule, requires the animal's attention to the external sensory input. The forebrain Ss may have performed more poorly than the hypothalamic Ss in the long discrimination session because of a cumulative inhibitory effect of the forebrain ESB on the sensory input necessary for the discrimination. The assumption here is that the forebrain ESB does not have a significant effect on the sensory input at the beginning of the session but only later, as a cumulative effect, perhaps a biochemical one.

However, this hypothesis, that forebrain ESB has a long-term inhibitory effect on sensory input, may not be sufficient to explain the performance of the hypothalamic Ss in the discrimination session. All of the hypothalamic Ss responded for many hours without pausing for longer than $5 \mathrm{~min}$; two of them responded without pauses for the entire 48-h session. The hypothalamic ESB must have had some positive effect, besides not producing long-term inhibition of sensory input, to keep the animals going. The notion that hypothalamic ESB has an antifatigue effect, which was earlier given to explain the differences in onset of pauses, would seem to apply here; in the discrimination situation, where the physical factors of fatigue are considerably reduced, the effect of the hypothalamic ESB on motor facilitation or general arousal can emerge more fully.

At first glance, it would seem that this last assumption concerning the occurrence of natural fatigue makes superfluous the hypothesis of reduced sensitivity to sensory input in forebrain animals. It may be argued that the poor performance of forebrain animals was simply due to fatigue, and the good performance of hypothalamic Ss was a result of the facilitatory or arousing effects of hypothalamic ESB on performance. However, two points make the hypothesis in question necessary to account for the behavior of the forebrain Ss. First of all, if poorer performance was simply due to fatigue, then forebrain Ss should return after a rest period to the initial level of performance; the endurance scores of the discrimination session show that this was not the case. Second, it is in the discrimination session, where the animal is required to respond only once per $\mathrm{S}+$ and where it is allowed to rest during the $\mathrm{S}-$ period, that forebrain Ss show poorer performance; in the CRF session, where the effects of fatigue should be considerably greater, "the endurance and decrement scores for the two groups did not differ significantly.

\section{REFERENCES}

Atrens, D. M. Reinforcing and emotional consequences of electrical self-stimulation of the subcortical limbic-forebrain. Physiology \& Behavior, 1970, 5, 1461-1471.

Ball, G. G. Electrical self stimulation of the brain and sensory 
inhibition. Psychonomic Science, 1967, 8, 489-490.

Christopher, S. M., \& Butter, C. M. Consummatory behaviors and locomotor exploration evoked from self-stimulation sites in rats. Journal of Comparative \& Physiological Psychology, $1968,66,335-339$.

Dafny, N., \& Feldman, S. Effects of stimulating reticular formation, hippocampus and septum on single cells in the posterior hypothalamus. Electroencephalography \& Clinical Neurophysiology, 1969, 26, 578-587.

Herberg, L. J., Tress, K. H., \& Blundell, J. E. Raising the threshold in experimental epilepsy by hypothalamic and septal stimulation and by audiogenic seizures. Brain, 1969, 92, 313-328.

Kent, E., \& Grossman, S. P. Evidence for a conflict interpretation of anomalous effects of rewarding brain stimulation. Journal of Comparative \& Physiological Psychology, 1969, 69, 381-390.

Lenzer, I. I., \& Frommer, G. P. Successive sensory discriminative behavior maintained by intracranial self-stimulation reinforcement. Physiology \& Behavior, 1968, 3, 345-349.

Lenzer, I. I., \& Frommer, G. P. Successive sensory discriminative behavior maintained by forebrain self-stimulation reinforcement. Psychonomic Science, 1971, 23, 88-90.

Olds, J. Satiation effects in self-stimulation of the brain. Journal of Comparative \& Physiological Psychology, 1958, 51, 675-678.

Ray, O. S., Hine, B., \& Bivens, L. W. Stability of self-stimulation responding during long test sessions. Physiology \& Behavior, 1968, 3, 161-164.

Reid, L. D., Gibson, W. E., Gledhill, S. M., \& Porter, P. B. Anticonvulsant drugs and self-stimulating behavior. Journal of Comparative \& Physiological Psychology, 1964, 57, 353-356.

(Received for publication August 28, 1972; accepted October 12, 1972.) 